EDITORIAL

\title{
Unanticipated rare adverse events and the surgeon startle response in ophthalmic surgery
}

(c) The Author(s), under exclusive licence to The Royal College of Ophthalmologists 2021, corrected publication 2021

Eye (2022) 36:3-4; https://doi.org/10.1038/s41433-021-01703-x

\section{EDITORIAL}

On January 15, 2009, Captain Sully Sullenberger successfully landed US Airways Flight 1549 on the Hudson River after the plane struck a flock of birds, losing all engine power. All aboard the flight survived, with few serious injuries. Here, he reflects on his startle response during the "Miracle on the Hudson" [1, 2].

"We plan ahead, anticipate every course of action, and have alternative courses of action. But the startle effect was huge in those first seconds ... My blood pressure shot up. My pulse spiked. We all got tunnel vision as our perceptual fields narrowed because of the stress. But, as professionals, we ... had the mental discipline to compartmentalize our minds and focus clearly on the tasks at hand" $[1,2]$.

The Federal Aviation Administration defines startle as an uncontrollable, automatic muscle reflex, raised heart rate, blood pressure, etc., elicited by exposure to a sudden, intense event that violates a pilot's expectations [3]. There are considerable cognitive effects on information processing following a startle event, leading to cognitive and dexterous impairment lasting up to $30 \mathrm{~s}$ [4]. All of us, as surgeons, face sudden adverse events during surgery: the posterior capsule breaks, intraocular contents jet out from the incision, or the eye suddenly becomes rock hard. These events can trigger the startle response: confusion, fear, narrowed focus, cognitive impairment if not paralysis, flushing, rapid heart rate, trembling hands, decreased motor skills, and impulsive actions.

Superimposed are feelings of disappointment and failure as we struggle to cope with the situation while picturing the patient's face the next day. Furthermore, surgeons may experience lasting physiologic and psychologic effects from the event, particularly when disclosure of error is not encouraged and debriefing is not done [5]. The authors could not identify any reference to startle in the ophthalmologic literature. We look to aviation literature analysis of this phenomenon and apply it to ophthalmologic intraoperative adverse events [6-8].

\section{PREPARATION AND ANTICIPATION}

A lack of training and/or experience impairs the surgeon's ability to manage rare adverse events. Furthermore, a lack of expectation of these events reduces the perceived necessity to be prepared. Both "deficits" increase the likelihood and magnitude of startle. Once an unusual or emergency situation is presented, a pilot or surgeon will generally be limited in their response to patterns the individual has seen before [9]. Anticipation of and preparation for rare adverse events help minimize, or even avoid, startle.
Residency and fellowship training programs should empower trainees to master management of unanticipated rare adverse intraoperative events and instil the concept that these skill sets need to be constantly refreshed. In clinical practice, these skills can sometimes be maintained through surgical experience. But often, for rare events, the surgeon must rely upon periodic review of educational resources (monographs, videos, continuing medical education, simulation of adverse events). Further, pre-operative planning and understanding of key patient risk factors, such as pseudo-exfoliation, can help mitigate the startle response.

A critical factor for both of these options is recency. In aviation, commercial airline pilots are required to maintain recency: making three take offs and landings in a rolling 90-day period in order to maintain certification (Title 14, Code of Federal Regulations, Part 61). It is similarly reasonable for surgeons to regularly review management protocols for rare events to mitigate startle. Maintenance of recency could depend on volume and years of experience, although startle can occur even in seasoned professionals e.g., Sully Sullenberger. Optimally, the surgeon can mentally review these protocols immediately before every operation to keep management fresh.

Intraoperatively, the best way to avoid startle is prevention: recognizing when you are in high-risk situation that may lead to an adverse event unless remedial action is taken (e.g. phaco'ing deep in the bag; lax zonules). Early recognition of the signs of adverse events (e.g. pupil snap, sudden deepening or shallowing of the anterior chamber, or increasing IOP) can empower the surgeon to mitigate the situation. At each stage, denial is a powerful factor, so it is imperative for the surgeon to not dismiss warning signs.

A key issue is the surgeons' initial reaction to an adverse event, which can be compromised by startle. Some events require immediate action (e.g. for a developing expulsive hemorrhage: close the incision as fast as possible). Others require restraint (e.g. resisting the temptation to pull a phaco tip filled with vitreous out of the eye, instead keeping it where it is and clear vitreous from the tip before withdrawing the instrument). All require level-headedness, gathering information ... situational awareness ... and a formulating an at times complex plan of action.

When an unexpected adverse event occurs, communication is paramount to ensuring everyone is aware of the situation and prepared to assist. Immediately alert anesthesia and the nursing staff, which could be done through coded language, such as "code

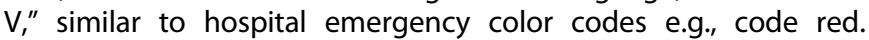
Essential information is thus conveyed while minimizing stress and alarm to the patient. It is crucial to put all team members on alert and they communicate back their actions. Creating and reviewing standardized protocols for these types of adverse events with nursing staff, just as we list preferences for operating room instruments, can aid response to adverse events, especially when there is staff turnover between and even during cases. 


\section{THE EMOTIONAL AFTERMATH}

Coping can be difficult: supporting and teaching each other are imperative. Understand that the reaction to the event during and afterwards is normal. We are all aware of the emotional toll of intraoperative adverse effects. Support your colleagues who have experienced intraoperative adverse events, and when you experience one, reach out to your support system [5, 10-13].

Startle is a surgical event that every surgeon can encounter at any point in a career. Knowing which situations may elicit startle and preparing for them on an ongoing basis will protect both patient and surgeon. Consideration should be given to enhanced emphasis on handling specific startle inducing situations and startle management in residency training programs and to maintenance of recency in clinical practice.

\section{DISCLAIMER}

The view(s) expressed herein are those of the author(s) and do not reflect the official policy or position of Walter Reed National Military Medical Center, the U.S. Army Medical Department, the U.S. Army Office of the Surgeon General, the Department of the Air Force, the Department of the Army, Department of Defense, the Uniformed Services University of the Health Sciences or any other agency of the U.S. Government.

$$
\text { Angela Chang }{ }^{1} \text {, Grant A. Justin } \mathbb{D}^{2,3} \text {, Priya M. Mathews }{ }^{4} \text { and }
$$
James D. Auran (iD ${ }^{1 \times}$

${ }^{1}$ Edward S. Harkness Eye Institute, Department of Ophthalmology, Columbia University Irving Medical Center, New York, NY, USA.

${ }^{2}$ Department of Ophthalmology, Walter Reed National Military Medical Center, Bethesda, MD, USA. ${ }^{3}$ Department of Surgery, Uniformed Services University of Health Science, Bethesda, MD, USA. ${ }^{4}$ Department of Ophthalmology, Johns Hopkins University School of Medicine, Baltimore, MD, USA. ${ }^{\varpi}$ email: jda5@cumc.columbia.edu

\section{REFERENCES}

1. Buchanan L. 10 Years After the 'Miracle on the Hudson,' Sully Sullenberger Talks Incredible Mental Discipline and How to Handle Pressure Inc. 2019. https://www. inc.com/leigh-buchanan/sully-sullenberger-leadership-lessons.html.
2. Sullenberger Discusses Startle Response. https://www.youtube.com/watch? $\mathrm{v}=$ Sc8f0jluRk0. Accessed May 2020.

3. FAA. Advisory Circular 120-111. Upset Prevention and Recovery Training. 2017. https://www.faa.gov/documentLibrary/media/Advisory_Circular/AC_120111_CHG_1_FAA_Editorial_Update.pdf. Accessed May 2021.

4. Thackray RI, Touchstone RM. Recovery of motor performance following startle. Percept Mot Skills. 1970;30:279-92.

5. Luu S, Leung SO, Moulton CA. When bad things happen to good surgeons: reactions to adverse events. Surg Clin North Am. 2012;92:153-61.

6. Singh A. Startle vs Surprise and flight crew training. 2019. https://safetymatters. co.in/2019/06/25/startle-vs-surprise-mindfly/.

7. Without warning: the startle factor Flight Safety Australia. 2015. https://www. flightsafetyaustralia.com/2015/08/without-warning-the-startle-factor/.

8. Flight Safety Foundation. Startle Effect. https://www.skybrary.aero/index.php/ Startle_Effect. Accessed May 2021.

9. Gillen M. A Study Evaluating if Targeted Training for Startle Effect can Improve Pilot Reactions in Handling Unexpected Situations in a Flight Simulator. https://commons.und.edu/theses/345: University of North Dakota; 2016.

10. Bohnen JD, Lillemoe KD, Mort EA, Kaafarani HMA. When Things Go Wrong: The Surgeon as Second Victim. Ann Surg. 2019;269:808-9.

11. Han K, Bohnen JD, Peponis T, Martinez M, Nandan A, Yeh DD, et al. The Surgeon as the Second Victim? Results of the Boston Intraoperative Adverse Events Surgeons' Attitude (BISA) Study. J Am Coll Surg. 2017;224:1048-56.

12. Sataloff RT. Adverse Surgical Events: Effects on the Surgeon. Ear Nose Throat J. 2020;99:225-6.

13. Thompson CV, Naumann DN, Fellows JL, Bowley DM, Suggett N. Post-traumatic stress disorder amongst surgical trainees: an unrecognised risk? Surgeon. 2017; 15:123-30.

\section{COMPETING INTERESTS}

The authors declare no competing interests.

\section{ADDITIONAL INFORMATION}

Correspondence and requests for materials should be addressed to J.D.A.

Reprints and permission information is available at http://www.nature.com/ reprints

Publisher's note Springer Nature remains neutral with regard to jurisdictional claims in published maps and institutional affiliations. 